\title{
Actualización en el tratamiento con soluciones tópicas no corticoesteroidales en patología rinosinusal. Revisión de la literatura
}

\author{
Update on non corticosteroidal topical solutions for rhinosinusal disease treatment. \\ Literature review
}

Eugenio Alzérreca $A^{1}$, Paul Boettiger $B^{2}$, Sebastián Olivares $\mathbf{M}^{3}$, Héctor Bahamonde $\mathrm{S}^{2}$, Alfredo Naser $\mathrm{G}^{2}$.

\section{RESUMEN}

La rinosinusitis es una enfermedad frecuente, con una morbilidad considerable y una repercusión significativa sobre la calidad de vida de los pacientes. Existe un conjunto de tratamientos tópicos complementarios en el manejo sintomático de la patología rinosinusal, dentro de los que se incluyen el uso de lavados nasales con diferentes soluciones. El objetivo de esta revisión fue evaluar los resultados en la literatura, de los diferentes métodos de aplicación de medicamentos tópicos nasales por irrigación y las diferentes soluciones utilizadas. Existen múltiples técnicas para la irrigación nasal, desde jeringas a presión positiva, hasta instilación por gravedad. Las soluciones se pueden categorizar en salinas, antibióticas, antifúngicas y detergentes, todas con indicaciones y resultados variados. La irrigación nasal con soluciones, representa una muy buena alternativa de tratamiento adyuvante en patología rinosinusal, al mejorar la calidad de vida de los pacientes, siendo un método de bajo costo de implementación y seguro.

Palabras claves: Rinosinusitis, irrigación nasal, tratamientos tópicos nasales.

\begin{abstract}
Rhino sinusitis disease is a common problem with considerable morbidity and a significant impact on patient's quality of life. There is number of adjuvant treatments to manage symptoms of sinus disease, which includes the use of nasal lavages with different solutions. The aim of this review was to evaluate the literature results of different methods of application and solutions used for nasal topical irrigation. There are many techniques for nasal irrigation, from syringes with positive pressure to gravity instillation. The solutions can be categorized into saline, antibiotic, antifungal and detergents, all with particular indications and multiple results. Nasal irrigation represents a good alternative of adjuvant therapy in rhino sinusitis, to improve our patient's quality of life with a low-cost and safe method.
\end{abstract}

Key words: Rhinosinusitis, Nasal Lavage, Nasal Topical Treatment.

\footnotetext{
'Médico Cirujano, Magíster en Derecho de la Salud.

${ }^{2}$ Servicio de Otorrinolaringología, Hospital Clínico de la Universidad de Chile.

${ }^{3}$ Interno Medicina, Universidad de Chile.
} 


\section{INTRODUCCIÓN}

La rinosinusitis es una enfermedad frecuente, con una morbilidad considerable, síntomas refractarios comunes y una repercusión significativa sobre la calidad de vida de los pacientes. El término de rinosinusitis hace referencia a un variado espectro de desórdenes inflamatorios que afectan concomitantemente a las cavidades paranasales como a la cavidad nasal. A partir de mediados de la década del 90, este término reemplazó a la denominación antigua de sinusitis, puesto que esta entidad aislada es rara, presentándose y usualmente siendo precedida por una rinitis ${ }^{1}$. Se ha propuesto un sistema de clasificación clínica de las rinosinusitis de acuerdo a su perfil de temporalidad sintomática, el cual las divide en rinosinusitis aguda, con una duración de hasta 4 semanas, en subaguda cuando la duración de los síntomas es entre 4 y 12 semanas y en rinosinusitis crónica (RSC) cuando los síntomas están presentes por más de 12 semanas ${ }^{1}$.

En USA, la RSC afecta entre $5 \%$ a $15 \%$ de la población general, reuniendo aproximadamente 26,7 millones de consultas anuales y resultando en 5,8 billones de dólares anuales de costo directo ${ }^{1}$. A nivel nacional, en el estudio de Ruz y $\mathrm{col}^{2}$, sobre patología otorrinolaringológica ambulatoria, la rinosinusitis aguda y RSC correspondieron al 3,6\% de las entidades revisadas en forma individual. Los pacientes con RSC han mostrado una calidad de vida similar a la de pacientes con artritis, cáncer, asma y síndrome de intestino irritable; además presentan un mayor índice de ausentismo laboral y de visitas a médicos de familias, medicina alternativa y salud menta|3,4.

El tratamiento de la RSC permanece como un tema de controversia considerable, que incluye como pilares principales la antibioticoterapia a corto y largo plazo y la utilización de corticosteroides tópicos y sistémicos, todo esto asociado a terapias adyuvantes como los descongestionantes tópicos y orales, antihistamínicos orales y uso de mucolíticos ${ }^{5,6}$.

Existe un conjunto de tratamientos con medicamentos tópicos que han demostrado ser de utilidad en el manejo sintomático de la patología rinosinusal y se han incluido como complemento en la mayoría de los protocolos de tratamiento en rinosinusitis. Este grupo de terapias incluye el uso de lavados o irrigación nasal con variadas preparaciones de soluciones para irrigación, que se diferencian tanto en su composición como en sus técnicas de aplicación, existiendo diversas presentaciones comerciales y caseras como aerosol, bombas o botellas de irrigación ${ }^{6}$.

La principal característica de la mucosa nasal es su epitelio pseudo estratificado de tipo ciliado, que mediante el clearence mucociliar fisiológico, favorece la distribución de los agentes farmacológicos utilizados, pero a la vez disminuye el tiempo de contacto directo del medicamento con la región a tratar ${ }^{7}$. La mucosa nasal presenta una superficie de $160 \mathrm{~cm}^{2}$ con un volumen aproximado de $0,4 \mathrm{ml}$, por lo que la cantidad de medicamento requerido para cubrir toda la mucosa es teóricamente pequeña ${ }^{7}$. A pesar de esto, los lavados nasales, en especial aquellos con soluciones salinas, son considerados con frecuencia como un complemento alternativo del tratamiento médico de las enfermedades nasosinusales.

Se ha propuesto que la base de su beneficio sintomático, sería el arrastre mecánico de las secreciones sinusales, sin embargo, se ha evidenciado su efecto en la mejoría de la motilidad del epitelio ciliar sinusal, así como su capacidad de extracción de antígenos, un efecto en disrupción de biofilms en el caso de aquellas soluciones de tipo detergente, y una función protectora de la mucosa ${ }^{7}$.

La mayoría de los estudios randomizados controlados existentes sobre el tema, se han hecho en grupos de pacientes con RSC en poblaciones pequeñas y sin una estandarización en la medición de resultados, por lo que la efectividad del tratamiento de la enfermedad nasosinusal con irrigación mediante soluciones, permanece siendo una incógnita hasta hoy ${ }^{6}$. En nuestro país, no existen estudios controlados sobre la materia aunque en la práctica, estos tratamientos son utilizados con frecuencia en los diferentes centros de atención primaria y de especialidad.

En esta revisión nos enfocaremos en la utilización de las diferentes soluciones no corticosteroidales existentes para irrigación nasal, como herramienta para el tratamiento y manejo sintomático en la patología rinosinusal. Los diferentes niveles de evidencia de los estudios revisados se detallan en la Tabla 1. 
Tabla 1. Niveles de evidencia científica

\begin{tabular}{|ll|}
\hline $\begin{array}{l}\text { Niveles de } \\
\text { evidencia científica }\end{array}$ & Graduación de los diseños de estudio \\
\hline I & Evidencia a partir de ensayos clínicos aleatorios \\
II-a & Evidencia a partir de ensayos clínicos no aleatorios \\
II-b & Evidencia a partir de estudios de cohorte o casos y controles, realizados por más \\
III & de un centro \\
IV & Evidencia a partir de comparaciones en el tiempo o entre sitios. Puede incluir \\
& resultados de estudios no aleatorios \\
& expertos de expertos, basado en la experiencia clínica. Informes de comités de \\
\hline
\end{tabular}

Calidad de diseño y rigor cientifico de mayor (I) a menor (IV). De: US. Preventive Task Force. Guide to clinical preventive services: An assessment of the effectiveness of 169 interventions. Baltimore: Williams and Wilkins, 1989.

\section{MÉTODOS DE DISTRIBUCIÓN DE LAS SOLUCIONES}

Las técnicas de irrigación o lavados nasales han tenido una evolución importante durante la historia, tanto en las características de los diferentes métodos de aplicación en la cavidad nasal y cavidades para nasales, como en la naturaleza y composición de las soluciones utilizadas en ellos. Las soluciones tópicas nasales pueden ser aplicadas a través de diversas prácticas, como la utilización de presión positiva por aerosol, pulsos o duchas, 0 por gravedad utilizando distintos tipos de surtidores $^{8}$.

Un estudio de Olson y col' ${ }^{8}$, evalúo la distribución de soluciones salinas isotónicas mediante irrigaciones con $40 \mathrm{ml}$ de medio de contraste no iónico, en pacientes sin historia de enfermedad aguda o crónica rinosinusal, desviación septal sintomática ni alergias, inmediatamente antes de realizar una tomografía computarizada para visualizar cavidades paranasales. Para cada sujeto de estudio se utilizaron 3 métodos de irrigación: uno con presión positiva de irrigación, uno con presión negativa e irrigación utilizando un nebulizador. El resultado obtenido evidenció que tanto los métodos con presión positiva y negativa lograban una distribución de contraste principalmente a los senos maxilares y etmoidales, a diferencia del método de nebulización que presentó una distribución pobre y poco uniforme de contraste a las cavidades paranasales $(p<0,05)$ (Tabla 2).

\section{TIPOS DE SOLUCIONES}

Las diferentes soluciones utilizadas en irrigación nasal, se pueden dividir según su composición de agentes farmacológicos activos en 4 grandes grupos: soluciones salinas, soluciones antibióticas, soluciones antifúngicas y soluciones detergentes. Fuera de esta categorización propuesta para definir los distintos tipos de soluciones usadas, existen múltiples composiciones farmacéuticas comerciales que incluyen agentes antiinflamatorios 0 antihistamínicos en la soluciones.

A continuación, revisaremos de acuerdo a esta clasificación, las diferentes soluciones existentes y sus principales resultados en estudios clínicos.

\section{Soluciones salinas}

Las soluciones salinas utilizadas en irrigación nasal actúan mediante un baño de la cavidad nasal al instilar la solución salina por una fosa nasal y permitiendo su drenaje por la fosa contralateral. Típicamente se describen 2 tipos de soluciones salinas: al 0,9\% y al 3\%, isotónicas e hipertónicas, respectivamente. Sin embargo, la concentración óptima, el pH y la temperatura adecuada son 
Tabla 2. Métodos de distribución de soluciones salinas en irrigación

\begin{tabular}{|llrl|}
\hline Métodos & \multicolumn{1}{c|}{ Dósis } & $\% \mathrm{NaCl}$ & Preparación \\
\hline Pera & $150 \mathrm{ml}$ c/12 hrs. & $2 \%$ & 1 cda. sal, $1 / 2 \mathrm{cda}$. bicarbonato sodio, $480 \mathrm{ml}$ agua \\
Atomizador & 4 puff c/8 hrs. & $0,9 \%$ & Agua de mar \\
Jeringa & $250 \mathrm{ml} \mathrm{c} / 12 \mathrm{hrs}$. & $3 \%$ & 3 cdas. de sal, 1 cda. de bicarbonato de sodio, $950 \mathrm{ml}$ agua \\
Botella a presión & $200 \mathrm{ml} / 12 \mathrm{hrs}$. & $0,9 \%$ & 1 cda. sal, 1 cda bicarbonato sodio, $500 \mathrm{ml}$ agua. \\
\hline
\end{tabular}

desconocidas y pueden variar en cada paciente. Los lavados nasales utilizando solución salina son útiles para el manejo sintomático de la RSC, siendo ésta la indicación más común para esta técnica ${ }^{12}$.

En un estudio de Rabago y $\mathrm{col}^{13}$, se determinó el efecto de lavados diarios con solución salina al $2 \%$ como terapia adyuvante a la de rutina en RSC, durante 6 meses, en pacientes con RSC posoperados mediante cirugía endoscópica funcional (CEF). Se obtuvo una reducción del $64 \%$ de la severidad de los síntomas según las escalas de evaluación subjetiva Medical Outcomes Survey Short Form (SF-12, Encuesta Abreviada de Resultados Médicos) y el Rhinosinusitis Disability Index (Índice de discapacidad en rinosinusitis). Estos pacientes además experimentaron una significativa mejoría en su calidad de vida a los 6 y 18 meses de seguimiento.

Existe un rango de otras condiciones como rinitis, rinosinusitis aguda 0 el embarazo, que pueden presentar respuesta a las irrigaciones nasales con solución salina. En un estudio de Slapak y col'12, se evaluaron 390 pacientes pediátricos con rinitis que recibieron tratamiento adyuvante con lavados nasales de solución salina isotónicos versus un grupo control sin irrigación nasal. Se evidenció una mejoría sintomática en el grupo de pacientes irrigados con solución salina, en términos de obstrucción nasal $(p<0,05)$, evaluada mediante una escala de 4 puntos, donde 1 corresponde a flujo aéreo sin dificultad y 4 a obstrucción completa. Se evalúo también el tipo de secreciones nasales presentes, dividiéndolas entre ausentes, serosas, seropurulentas 0 purulentas, obteniendo una diferencia significativa entre ambos grupos de pacientes, con una predominancia de secreciones serosas 0 ausencia de ellas en el grupo de pacientes irrigados $(p<0,05)$. Por último, se incluyó una escala de bienestar general sintomático, calculada en base a 4 puntos sobre el grado de mejoría en el examen clínico, donde 1 representa la remisión sintomática y 4 la persistencia en los síntomas. El puntaje promedio del grupo control fue de 2,06 (95\% IC 1,93-2,19) comparado con 1,72 (95\% IC 1,66-1,78) del grupo que recibió irrigaciones con solución salina isotónica.

Las modificaciones a la técnica y salinidad de la solución pueden ayudar a evitar efectos adversos menores de la irrigación salina, como sensación de malestar, ardor nasal, irritación o náuseas. De acuerdo a una revisión de Cochrane ${ }^{14}$, de entre 1.659 pacientes obtenidos de 22 ensayos clínicos, menos del $10 \%$ registraron alguna reacción adversa a la técnica, y no se registraron eventos adversos importantes con el uso de solución salina hipertónica e isotónica.

Las contraindicaciones para la irrigación nasal en general, y de aquellas con solución salina incluyen trauma facial, por el riesgo potencial de contaminación de otras regiones vecinas, y condiciones asociadas con un mayor riesgo de aspiración, como patología neurológica o problemas músculo esqueléticos ${ }^{15}$.

Los niveles de evidencia y recomendación científica para soluciones salinas se expresan en la Tabla 3.

\section{Soluciones antibióticas}

La mayor cantidad de información disponible en la actualidad, sobre el uso de irrigaciones nasales con soluciones antibacterianas, se centra en la utilización de aminoglicósidos como gentamicina 0 tobramicina. La gentamicina se ha utilizado en 
Tabla 3. Recomendaciones sobre uso de soluciones topicas no corticoisteroidales en irrigación nasal

\begin{tabular}{|lccl|}
\hline Solución & Evidencia & Recomendación & Comentarios \\
\hline Salina & lb & D & $\begin{array}{l}\text { Recomendación como terapia adyuvante sintomática en pa- } \\
\text { cientes con RSC y post CEF. }\end{array}$ \\
Antibiótica & Ib & D & $\begin{array}{l}\text { Recomendación en pacientes con síntomas de RSC recurren- } \\
\text { tes y CEF previa. }\end{array}$ \\
Fúngica & Ila & D & $\begin{array}{l}\text { Recomendación en pacientes con síntomas de RSC y sospe- } \\
\text { cha de infección fúngica. } \\
\text { Recomendación en pacientes con síntomas de RSC recurren- } \\
\text { tes, post CEF y con secreción mucosa importante. }\end{array}$ \\
\hline
\end{tabular}

Niveles de recomendación. A: Basada en una categoría de evidencia I. Extremadamente recomendable. B: Basada en una categoría de evidencia II. Recomendación favorable. C: Basada en una categoría de evidencia III. Recomendación favorable pero no concluyente. D: Basada en una categoría de evidencia IV. Consenso de expertos, sin evidencia adecuada de investigación.

irrigación nasal a dosis de $80 \mathrm{mg}$ en $500 \mathrm{ml}$ de suero salino y la tobramicina se ha administrado en dosis de $20 \mathrm{mg}$ en $50 \mathrm{ml}$ de suero salino. La concentración utilizada debe estar basada sobre la premisa de que toda la droga será absorbida, de forma que la usual dosis sistémica no sea excedi$\mathrm{da}^{16}$. En una revisión realizada por Moss y $\mathrm{col}^{18}$, se describe el uso de una solución compuesta por neomicina $40 \mathrm{mg} / \mathrm{L}$ y polimixina B $200.000 \mathrm{U} / \mathrm{L}$, desarrollada originalmente para irrigación vesical. En su descripción, esta solución fue diluida al 50\% con suero salino y administrada como irrigación nasal en dosis de $50 \mathrm{ml}$, para con posterioridad obtener niveles plasmáticos a intervalos de 15 minutos, sin evidenciar absorción sistémica hasta 1 hora después del procedimiento.

En el mismo artículo de Moss y $\mathrm{col}^{18}$, se estudió el uso combinado de CEF más irrigaciones nasales con tobramicina, en dosis de $40 \mathrm{mg}, 3$ veces al día por 10 días, en el tratamiento de 32 pacientes con fibrosis quística más RSC, demostrando una reducción en los casos de requerimiento de nuevas CEF a 2 años de seguimiento.

A pesar de que las soluciones con gentamicina o tobramicina son excelentes elecciones cuando el cultivo sinusal evidencia la presencia de pseudomonas, serratia u otro organismo sensible a aminoglicósidos, está menos claro qué antibiótico tópico administrar para el tratamiento óptimo de bacterias Gram positivas, anaerobias, u otro organismo no sensible a aminoglicósidos. Basado en la literatura de patología broncopulmonar, se postula que la clindamicina podría ser una buena alternativa por su cobertura, especialmente cuando es combinada con corticosteroides tópicos ${ }^{16,17}$.

Existe un grupo de pacientes con RSC y síntomas persistentes, que normalmente se encuentran colonizados por Stafilococcus aureus, organismo conocido por producir toxinas que tienen la capacidad de actuar como superantígenos. En este grupo de pacientes se ha estudiado el efecto de la irrigación nasal con mupirocina al $0,05 \%$ por 3 semanas, logrando una concentración efectiva substancialmente mayor que la concentración inhibitoria mínima (CIM 0,12 ug/lt), siendo una terapia efectiva en el tratamiento de RSC asociada a colonización por Stafilococcus aureus ${ }^{19}$.

En relación al uso de irrigaciones con antibioticoterapia en CEF, Leonard y $\mathrm{col}^{6}$, realizaron un estudio utilizando irrigaciones nasales con ceftazidima ( $300 \mathrm{mg}$ en $300 \mathrm{ml}$ de solución salina) en pacientes con RSC con síntomas recurrentes y CEF previa, evidenciado una mejoría sintomática de acuerdo a la escala SNOT-20 con buena tolerancia a este tratamiento.

Los niveles de evidencia y recomendación científica para soluciones antibióticas se expresan en la Tabla 3.

\section{Soluciones antifúngicas}

Existe una alteración en la respuesta inmunitaria local frente a la presencia de hongos en las 
secreciones nasales, lo que determina la aparición de RSC eosinofílica. El estudio histopatológico de esta lesión normalmente demuestra presencia de hifas en el moco, con hallazgo de eosinófilos circundantes, como parte de la respuesta inflamatoria local. La infección por estos organismos tiene tendencia a ser indolente y de tratamiento complejo, que puede incluir en casos de invasión fúngica, cirugía para biopsia y debridación, asociado al uso de agentes antifúngicos sistémicos como anfotericina $\mathrm{B}$, que lamentablemente ha demostrado una efectividad $y$ pronóstico poco auspiciosos ${ }^{20}$. Estas características han llevado a plantear la posibilidad de tratar con antimicóticos tópicos a estos pacientes.

Ponikau y col$^{20}$, realizaron un estudio randomizado controlado en pacientes con RSC para determinar la eficacia del tratamiento con anfotericina $B$ en una dosis de $20 \mathrm{ml}$ en cada fosa nasal a una concentración de $250 \mathrm{mg} / \mathrm{ml}$ por 6 meses. A esta dosis, el estudio evidenció una reducción en el engrosamiento inflamatorio, según imágenes de tomografía computarizada y endoscopía nasal, y un descenso en las concentraciones de marcadores intranasales de inflamación eosinofílica, sin diferencia significativa en la reducción de IL-5.

Acerca del uso de irrigaciones nasales con terapia antifúngica en CEF, Riccheti y $\mathrm{col}^{21}$, investigaron la eficacia de lavados nasales con anfotericina $B$ en pacientes con poliposis nasal tratada con CEF previa, usando $1 \mathrm{gr}$. de anfotericina $B$ disuelto en $1.000 \mathrm{ml}$ de agua destilada, 2 veces al día por 4 semanas. Este estudio obtuvo como resultado la resolución completa de los pólipos en 39\% de los pacientes sin historia previa de CEF y en $47 \%$ de los pacientes con historia anterior de CEF.

Los niveles de evidencia y recomendación científica para soluciones antifúngicas se expresan en la Tabla 3.

\section{Soluciones detergentes}

Se ha estudiado mediante modelos in vitro e in vivo, la presencia de estructuras complejas tridimensionales llamadas biofilms, que alojan microorganismos bacterianos y les confieren resistencia a los tratamientos antibióticos convencionales, jugando un rol fundamental en la fisiopatología de la $\mathrm{RSC}^{22}$
Una de las alternativas de tratamiento que se han estudiado para la disrupción de biofilms, es la utilización de lavados nasales mediante shampoo de bebé al $1 \%$, que gracias a su contenido de agentes surfactantes, como PEG-80, actúa como detergente, disminuyendo la viscosidad y tensión superficial de las secreciones mucosas, evitando su unión al epitelio ciliado y favoreciendo de esta manera su eliminación mecánica por irrigación ${ }^{23}$.

Chiu y $\mathrm{Col}^{23}$, estudiaron los efectos in vitro del shampoo de bebé al $1 \%$ en biofilms de pseudomonas y en pacientes con RSC de difícil manejo, que permanecían sintomáticos pese al tratamiento médico y quirúrgico adecuado. Se utilizaron irrigaciones nasales con shampoo de bebé al 1\% $\left(\right.$ Johnson $\left.^{\circledR}\right)$ observando una mejoría sintomática objetiva en la escala SNOT-22 en un $50 \%$ de los casos, con un mayor beneficio evidenciado en pacientes con descarga mucosa importante.

Los niveles de evidencia y recomendación científica para soluciones detergentes se expresan en la Tabla 3.

\section{DISCUSIÓN}

Existe una gran variabilidad entre los diferentes estudios sobre irrigación nasal revisados. En primera instancia hay que diferenciar entre las distintas técnicas de aplicación de los medicamentos tópicos en fosa nasal y cavidades paranasales, que van desde la irrigación a presión positiva, hasta la instilación por gravedad de las soluciones a utilizar, y las diferentes soluciones existentes para ser administradas, sin un consenso claro en las indicaciones de cada una. Estos hechos, sumado a la falta de estudios con ensayos controlados randomizados impiden conocer en profundidad los beneficios de cada técnica de administración.

La irrigación con soluciones salinas se ha utilizado como parte adyuvante del tratamiento de las RSC hace bastante tiempo, con resultados variados entre autores. Un aspecto promisorio e importante de su uso es la buena tolerancia descrita en la literatura, ya sea con soluciones salinas isotónicas, como con soluciones hipertónicas. Heatley y $\mathrm{col}^{24}$, informaron sobre la opinión de los pacientes que utilizaron soluciones salinas, donde 
el $70 \%$ consideraban ventajoso su uso y las recomendarían a otros pacientes. Para la mayoría de los pacientes evaluados en los diversos estudios, el efecto beneficioso de las soluciones salinas parece superar sus inconvenientes, sin embargo, la evidencia al respecto es limitada por lo que no es posible por el momento recomendar la irrigación con solución salina como parte estándar del tratamiento en rinosinusitis.

Con respecto al uso de soluciones con antibióticos para irrigación nasal, se debe tener en consideración que su uso indiscriminado puede llevar al desarrollo de mecanismos de resistencia antibacteriana y efectos adversos como reacciones de hipersensibilidad ${ }^{6}$. Indudablemente se requiere de nuevos estudios para comprender la forma en que la mucosa nasal interactúa con los medicamentos tópicos, e idealmente desarrollar soluciones con agentes de propiedades antimicrobianas específicas, lo que podría revolucionar el tratamiento de la patología infecciosa rinosinusal.

Existen resultados conflictivos en el tratamiento tópico nasal con antifúngicos, lo que puede explicarse por las diferencias utilizadas en las dosis de las soluciones administradas en los distintos estudios, y en las preparaciones de las mismas. En ese sentido, es necesario el desarrollo de nuevos ensayos clínicos cuyos resultados permitan crear un consenso en la materia. En los diferentes estudios de Ponikau y $\mathrm{Col}^{20}$, se ha utilizado anfotericina B como terapia antimicótica tópica, sin embargo se debe tener presente que corresponde a un fármaco citotóxico y su aplicación durante un periodo prolongado puede ejercer efectos sistémicos.

Es importante destacar la utilización de shampoo de bebé al $1 \%$ como agente detergente en lavados nasales. Este corresponde a un producto disponible ampliamente en el mercado, de bajo costo y excelente tolerancia, por lo que su aplicación como terapia adyuvante en casos de RSC, sobre todo en pacientes posoperados de CEF y con secreciones abundantes, no debe ser despreciada ${ }^{23}$.

\section{CONCLUSIONES}

Se revisaron los distintos métodos de aplicación y soluciones nasales tópicas no corticosteroidales existentes, las cuales fueron categorizadas en soluciones salinas, antibióticas, antifúngicas y detergentes, cada una de las cuales presenta diferentes características e indicaciones particulares.

La administración de tratamientos tópicos nasales es una herramienta terapéutica importante en el manejo sintomático de pacientes con enfermedad rinosinusal. Uno de los aspectos más importantes de este conjunto de tratamientos, es su enorme potencial en lograr mejoría en la calidad de vida de los pacientes, lo que sumado a su bajo costo de implementación y seguridad representa una muy buena alternativa de tratamiento.

\section{BIBLIOGRAFÍA}

1. Thomas M, Yawn B, Price D. EPOS Primary Care Guidelines: European Position Paper on the Primary Care Diagnosis and Management of Rhinosinusitis and Nasal Polyps 2007. Prim Care Respir J 2008; 17: 79-89.

2. Ruz S, Breinbauer $H$, Arancibia M. Análisis epidemiológico de la patología otorrinolaringológica ambulatoria en el Hospital San Juan de Dios. Rev Otorrinolaringol Cir Cabeza CueIlo 2009; 69: 227-32.

3. Sturgess J, Chao J, Wong J. Cilia with defective radial spokes; a cause of human respiratory disease. N Engl J Med 1979; 300: 53-6.

4. Desrosiers M, Gerald A, Keith P. Canadian clinical practice guidelines for acute and chronic rhinosinusitis. Vital Health Stat 1995; 261-520.

5. Macdonald K, McNally J, Massoud E. The health and resource utilization of Canadians with chronic rhinosinusitis. Laryngoscope 2009; 119: 184-9.

6. FoKkens W, Lund V, Mullol J. European Position Paper on Rhinosinusitis and Nasal Polyps 2007. Rhinology 2007; 1-139.

7. Leonard D, Bolger W. Topical Antibiotic Therapy for Recalcitrant Sinusitis. Laryngoscope 2009; 109: 668-70.

8. OLSON D, RAsGon M. Radiographic Comparison of Three Methods for Nasal Saline Irrigation. Laryngoscope 2002; 112: 1394-7. 
9. Anand VK, et al. Epidemiology and economic impact of rhinosinusitis. Ann Otol Rhinol Laryngol Supp/ 2004; 193: 3-5.

10. Leung $R$, Katial $R$. The diagnosis and management of acute and chronic sinusitis. Prim Care 2008; 35: 11-24.

11. Joe $S$, Thambi R, Huang J. A systematic review of the use of intranasal steroids in the treatment of chronic rhinosinusitis. Otolaryngol Head Neck Surg 2008; 139: 340-7.

12. Slapak I, Skoupa J, Strnad P. Efficacy of isotonic nasal wash (seawater) in the treatment and prevention of rhinitis in children. Arch Otolaryngol Head Neck Surg 2008; 134(1): 67-74.

13. Rabago D, Zgierska A, Mundt M. Efficacy of daily hypertonic saline nasal irrigation among patients with sinusitis: A randomized controlled trial. Journal of Family Practice 2002; 51: 1049-55.

14. Harvey R, Hannan S, Badia L. Nasal saline irrigations for the symptoms of chronic rhinosinusitis. Cochrane Database of Systematic Reviews 2007, Issue 3. Art. No.: CD006394.

15. BARCLAY L. Use of Saline Nasal Irrigation Reviewed. Am Fam Physician 2009; 80: 1117-9.

16. Hong GoH Y, Richard L. Current Status of Topical Nasal Antimicrobial Agents. Laryngoscope 2000; 110: 875-80.

17. Elledge E, Whiddon R, Fraker J. The effects of topical oral clindamycin antibiotic rinses on the bacterial content of saliva on healthy human subjects. Otolaryngol Head Neck Surg 1991; 105: 836-9.

18. Moss R, King V. Management of sinusitis in cystic fibrosis by endoscopic surgery and serial antimicrobial lavage: reduction in recurrence requiring surgery. Arch Head Neck Surg 1995; 121: 566-72.

19. Brent U, Alkis P, Wormald P. Nasal Lavage With Mupirocin for the Treatment of Surgically Recalcitrant Chronic Rhinosinusitis. Laryngoscope 2008; 118: 1677-80.

20. Ponikau J, Sherris D, Weaver A. Treatment of chronic rhinosinusitis with intranasal amphotericin B: a randomized, placebocontrolled, double blind pilot trial. J Allergy Clin Inmunol 2005; 115: 125-31.

21. RicchettI A, Basile N, LANDis M. Effect of antifungal nasal lavage with amphotericin $B$ on nasal polyposis. J Otolaryngol 2002; 116: 2613.

22. Palmer JN. Bacterial biofilms: Do they play a role in chronic sinusitis? Otolaryngol Clin North Am 2005; 38: 1193-201.

23. Chiu A, Palmer J, Woodworth B. Baby shampoo nasal irrigations for the symptomatic postfunctional endoscopic sinus surgery patient. Am J Rhinol 2008; 22: 34-7.

24. Heatley D, McConnell K, Kille T. Nasal irrigation for the alleviation of sinonasal symptoms. Otolaryngol Head Neck Surg 2001; 125: 44-8. 